\title{
Obesity Related Issues
}

\author{
Jonida Haxhiu
}

\author{
Institute of Public Health, Rruga "Aleksandër Moisiu" nr 80, Tiranë, Albania
}

Email: jonidahaxhiu@yahoo.com

\section{Doi:10.5901/ajis.2014.v3n6p149}

\begin{abstract}
Obesity is a major problem in many countries. Problems that surrounds it includes a number of consequences including health, social, or psychological. Psychological consequences, unless make it difficult to overcome the situation, but on the other hand can also be incentive to further aggravated. This is a qualitative research. This is because it is thought that so, can better understand obesity, and especially psychosocial problems associated with it. Were conducted in-depth 8 interviews, with obese people, in the form of case study. It is also developed a focus group with obese people. Women have a greater social pressure than men to be elegant. Women also reported higher levels than men about the lack of satisfaction about their image. So it is not surprising that women have more negative psychosocial consequences of obesity than men. In eight of the participants in the study admit that actually or previously have been involved in binge episodes. They report that as this episode as e stressful one. Studies show that obese people generally have a low level of self-esteem. 3 participants in the study were obese called extreme obese.
\end{abstract}

Keywords: obesity, stress, overeating episodes, gender

\section{Introduction}

Obese persons resulting, in most cases with a variety of consequences in terms of psychological problems and not only. Friedman and Brownell concluded that obesity in most cases is associated with depressive symptoms, thus causing symptoms. Also, studies have shown that obese people have reported higher levels of stress experienced during the day. Also the anxiety they experience, it results to be substantially more disaggregated high. According Bruene, persons seeking treatment for obesity, experience higher levels of psychological problems, compared with obese persons who do not seek treatment.

While some obese individuals experience depression and related complications, most obese people appear to have essentially normal psychological functioning. These individuals, however, may have physical or psychosocial complications of their excess weight that detract from their well-being, even if their problems do not rise to the level of a psychiatric diagnosis.

Health-related quality of life (HRQL) is commonly defined as the impact of an illness on one's physical and mental functioning. Studies have consistently demonstrated that obese individuals have lower HRQL (i.e., greater impairment due to illness) than do average-weight individuals. Furthermore, HRQL varies within the obese population; it declines as the severity of obesity increases. BMI is more strongly and more consistently associated with the physical domains of HRQL than with those related to mental health.

Body image dissatisfaction has become so prevalent in America as to be described as a "normative discontent." Nearly half of women in the general population give a generally negative evaluation of their appearance. The results of one survey suggest that, from 1972 to 1997, the percentage of women dissatisfied with their overall appearance more than doubled (from 25 to 56\%). The percentage of men who were dissatisfied with their appearance nearly tripled (from 15 to 43\%) during the same time. The most commonly cited sources of dissatisfaction were weight and waist/abdomen for both women and men. Dissatisfaction appears to be greatest among Caucasians and among individuals who have internalized sociocultural appearance standards regardless of ethnicity.

Binge eating and psychopathology. Two of three studies found significantly more depressive symptoms in obese patients with BED, as determined by interview, than in obese nonbingers. Numerous other studies found that binge eaters, compared with nonbinge eaters, reported not only more symptoms of depression, but also lower self-esteem, greater symptoms of borderline personality disorder, and greater lifetime prevalence of any Axis I mental disorder, including substance abuse or dependence. The presence of binge eating, therefore, can be considered a risk factor for 
depression and various other forms of psychopathology.

At least two studies have met these requirements. Istvan et al. examined the relationship between BMI and depression, as measured by the Center for Epidemiologic Studies Depression (CES-D) scale, in a nationally representative sample of 32,000 adults aged 25-74 years. They found no relationship between BMI and CES-D scores among men. Women in the highest quintile of $\mathrm{BMI}\left(\mathrm{BMI} \geq 28.96 \mathrm{~kg} / \mathrm{m}^{2}\right)$, however, were $38 \%$ more likely to score in the depressed range on the CES-D than those who fell into the lower BMI quintiles. The relationship between BMI and CESD scores was stronger among women who smoked (in the past or at present) than women with no history of smoking.

Carpenter et al. similarly studied a large, nationally representative sample (of more than 40,000 people) and found that the relationship between obesity and depression varied by sex. Using a structured interview, the investigators found that obese men (those with a BMI $\geq 30 \mathrm{~kg} / \mathrm{m}^{2}$ ) were significantly less likely to report a history of major depression, suicidal ideation, or suicide attempts in the past year than men of average weight (BMI $\left.=20.8-29.9 \mathrm{~kg} / \mathrm{m}^{2}\right)$. By contrast, underweight men (BMI $\left.<20.8 \mathrm{~kg} / \mathrm{m}^{2}\right)$ were at a $25 \%$ increased risk for depression, $81 \%$ increased risk for suicidal ideation, and $77 \%$ increased risk for suicide attempts, compared to average-weight men. A different pattern was observed among women. Obese females were 37\% more likely than their average-weight peers to have experienced major depression in the past year. Obese women also were $20 \%$ more likely to report suicidal ideation and $23 \%$ more likely to have made a suicide attempt in the past year. For women, being underweight was not associated with depression or suicidality.

Studies of clinical populations. Prevalence estimates of psychopathology are typically higher in clinical samples of obese people than in the population at large. In uncontrolled studies of individuals seeking weight reduction, the lifetime prevalence of depressive disorders (e.g., major depression, dysthymia) ranged from 9.2 to $47.5 \%$, and the lifetime prevalence of other Axis I mental disorders (i.e., clinical syndromes) ranged from 2.5 to $31 \%$.

Lifestyle modification consisting of a low-calorie diet, increased physical activity, and group behavior therapy is probably the most studied intervention for weight loss. Reviews indicate that lifestyle modification typically produces a loss of $8-10 \%$ of initial weight in 20-26 weeks. Unfortunately, most individuals return to their baseline weight within 3-5 years of completing treatment. Significant regain also has been observed following very-low-calorie diets and even some forms of bariatric surgery (i.e., vertical banded gastroplasty). Contrary to expectations, however, weight loss and regain (i.e., weight cycling) does not appear to be associated with clinically significant depression. Cross-sectional studies that found no differences in depression between individuals with and without a history of weight cycling have been criticized for methodological limitations. More rigorous longitudinal studies, however, have yielded similar conclusions. For example, Foster et al. found that obese women who lost an average of $21.1 \mathrm{~kg}$ after 6 months of treatment but were 3.6 $\mathrm{kg}$ above baseline 5 years post-treatment exhibited significant improvements in mood. Mean scores on the Beck Depression Inventory (BDI) dropped from 12.7 to 9.3 at follow-up. These women also reported significant reductions in hunger and disinhibition of eating.

While studies suggest that weight cycling does not result in clinically significant depression, it is associated with decreased satisfaction with appearance, self-esteem, and self-confidence. The generally favorable finding that weight cycling is unrelated to depression should not lead clinicians to minimize the shame and distress-if subclinical in severity —-that patients frequently report in response to a history of failed attempts to control their weight.

\section{Methodology}

This is a qualitative research. This is because it is thought that so, can better understand obesity, and especially psychosocial problems associated with it.

It were conducted 8-depth interviews with obese people, in the form of case study. It is also developed a focus group with obese people.

With regard to ethical issues, is taken permission to participants regarding their free will to be included in the study. Also, were provided them confidentiality and privacy.

\section{Results}

Women have a greater social pressure than men to be elegant. Women also reported higher levels than men about the lack of satisfaction about their image. So it is not surprising that women have more negative psychosocial consequences of obesity than men.

In eight of the participants in the study admit that actually or previously have been involved in binge eating 
episodes. They report that this episode stressed them so much.

participants report frustration and disappointment as a result of regaining some or all of their lost weight

This study shows that participants have a decreased level of quality of life, because of their obesity. Also, this study show that obese individuals, in general, have a low self-esteem level.

3 participants in the study were obese called extreme obese.

\section{Discussion}

Gender and obesity. Women have a greater social pressure than men to be elegant. Women also reported higher levels than men about the lack of satisfaction about their image. So it is not surprising that women have more negative psychosocial consequences of obesity than men. Carpenter and Istvan found that obese women are $38 \%$ more likely than normal weight women to develop depressive symptoms. Men in this study were not found any significant relation between mood and depression, or more accurately link was found only a few depressive symptoms. This can be clearly seen in the study participants, where women, all agree and it appears that depressive problems, even treated about them, compared with men participants who deny the presence of depressive problems.

If we refer to the seeking 'help' to reduce obesity will see that women are statistically at higher figures, they that are devoted to such centers.

Quality of life. Physical pain may have a major impact on HRQL in obese individuals. Osteoarthritis, knee pain, and back pain, in particular, are common among obese individuals and are positively associated with BMI. The presence of pain may account for much of the variance in the relationships between BMI and other aspects of HRQL. The experience of pain could mediate the relationship between BMI and psychological distress (e.g., depression) in extremely obese individuals, although this hypothesis has not been tested. All the participants, report that they experience various physical pains, a fact that creates problems in the quality of their life.

Binge episodes. Binge episodes are defined as the consumption of a large amount of food accompanied by a loss of control while eating. In individuals with this disorder it is noted 1 or 2 times a week, for at least 6 months, and which is very stressful. According to studies, the prevalence of this disorder in the general population ranges from 0.7 to $4 \%$. Four of the participants in the study admit that actually or previously have been involved in binge episodes. They report that as many how many stress created this episode. They say, that appeared suddenly a great desire to eat and despite efforts to eliminate or reduce it was impossible, until she turned in prevail and they became "victims" of their desire, eating large amounts of food for a long time short, usually hipercaloric foods such as sweets, fatty foods, fast food, etc..

Binge eating also appears to be associated with body image dissatisfaction. Obese bingers reported significantly greater dissatisfaction than did obese nonbingers; the increased dissatisfaction among bingers could not be explained by mood or BMI.

In most cases, subjects report feeling much worse after such an episode, even provoking vomiting for disposal of food consumed.

Binge episodes observed even among persons with type 2 diabetes.

Extreme obesity. In fact, four of the participants in the study were obese called extreme obese. According to the studies, which is confirmed by the participants in this study, obesity often includes people who have this problem in a vicious circle, which means that the this problem is associated with a range of effects such as stress or anxiety, which in turn, may stimulate the desire to eat. Also eating, in most cases is associated with negative feelings. Being obese is a condition which is present in the minds of these people whole of the time, which adds to the stress. Obesity so often is a condition that causes stress can the person tendence to eat, while this further aggravate their problem, thus turning them into extremely obese.

Depresive simptoms. Wadden et al. found that obese patients with a BMI $\geq 40 \mathrm{~kg} / \mathrm{m}^{2}$ had significantly greater depressive symptoms and significantly lower self-esteem than those with a BMI $<40 \mathrm{~kg} / \mathrm{m}^{2}$. These researchers suggested that the increased risk of medical complications and the greater likelihood of experiencing prejudice and discrimination might account for the greater depressive symptoms in obese patients. In general, participants in the study, confess that they experience depressive symptoms because they have to cope every moment with their obesity. Also, a continuous stress, increases the risk for health complications. Females experience more stress and depressive symptoms, because of obesity, than males.

Low self-esteem. Although obese people are more dissatisfied with their bodies than are their average-weight peers, body image dissatisfaction is moderately to strongly associated with greater depressive symptoms and lower selfesteem regardless of weight. The direction of the relationship between body image dissatisfaction and clinically significant 
depression is unknown. Research has yet to determine whether depressed individuals evaluate their bodies more negatively than do nondepressed people, whether dissatisfaction with appearance increases vulnerability to depression, or whether one or more other factors simultaneously increase the risk of both depression and body image dissatisfaction. Participants report body dissatisfaction, especially females. Also, participants report frustration and disappointment as a result of regaining some or all of their lost weight.

The earlier people become obese, the more likely they are to be dissatisfied with their bodies. Several studies have observed a relationship between being teased about weight in childhood and body image dissatisfaction in adulthood. Others, however, found that adulthood teasing was more strongly related to body image dissatisfaction than was childhood teasing. The relationship between recalled childhood teasing and body image may be partly explained by biases in memory due to current mood and self-esteem.

Obese individuals, in general, as indicated by the participants in the study, have a low self-esteem level. This for some reasons. First they think they are ugly, unattractive thing that makes think negatively about themselves, to have a low appreciation for it. Also, obese people, in most cases, have a low self-esteem due to the fact that they consider themselves to be powerless, to be they who would like, to lose weight and ranged in weight and body shape "dreamed". They think they are weak and they are unable to overcome this situation. Another factor that affects self-attribution is also the perceptions of others toward them. Obese people feel that others, in most cases, prejudice them due to their obesity, conceive unwilling to change, etc..

Although obese people are more dissatisfied with their body compared to the likes of normal weight, weight dissatisfaction strongly linked with depressive symptoms and low self-esteem. What is the type of relationship that which affects to other growth is still unknown.

\section{Conclusions}

This study has supported the common assumption that obesity is broadly associated with depression and other psychopathology. In men in particular, excess weight may be associated with a reduced risk of psychological complications. Conversely, being female, binge eating, and having a BMI $\geq 40 \mathrm{~kg} / \mathrm{m}^{2}$ increase the likelihood that a patient will report a significant mood disturbance. People who seek professional weight loss therapy also are more likely to be depressed than nontreatment-seeking obese individuals. Their mood disturbance may be one of the factors that prompts them to seek weight loss.

Obesity appeared a number of risks. It has physical and psychological risks.

Thus, obese persons, experience high levels of depressive symptoms.

Also, stress to them is high because all the time they present a situation that should start to change.

Binge episodes are another problem more pervasive among obese persons.

Also, the self-assessment, seen at very low levels to obese people, it's because of dislike of their bodies.

\section{Recommendations}

Obese people should have as much information about obesity, with related the consequences and appropriate ways to reduce it. This would enable a more suitable ground, to successfully cope with this condition and to make efforts to reduce it.

Also important is to get the information to the relatives of obese persons. Regarding this situation, the consequences and management methods will proceed successfully, which will enable a valuable and effective support for obese by persons, which will definitely reflect positively at an obese person.

Depression in obese people should not be assumed to be attributable solely to weight. Although losing weight does improve mood, clinicians should not expect patients' depression to remit with weight loss.

Treatment of the mood disorder with psychotherapy or pharmacotherapy should precede weight loss therapy. Alleviation of patients' psychological distress should improve their ability to adhere to diet and activity recommendations and, thus, achieve the weight loss they seek.

\section{References}

Norgan NG. The beneficial effects of body fat and adipose tissue in humans. Int J Obes Relat Metab Disord 1997;21: 738-746.

Wilson PW, D'Agostino RB, Sullivan L, Parise H, Kannel WB. Overweight and obesity as determinants of cardiovascular risk: the Framingham experience. Arch Intern Med 2002;162:1867-1872. 
Stevens J, Cai J, Pamuk ER, Williamson DF, Thun MJ, Wood JL. The effect of age on the association between body-mass index and mortality. N Engl J Med 1998;338:1-7.

Hughes D, McGuire A. A review of the economic analysis of obesity. Br Med Bull 1997;53:253-263.

National Task Force on the Prevention and Treatment of Obesity, National Institutes of Health. Very low-calorie diets. JAMA 1993;270:967-974.

Lee CD, Jackson AS, Blair SN. US weight guidelines: is it also important to consider cardiorespiratory fitness? Int J Obes Relat Metab Disord 1998;22(Suppl 2):S2-S7.

Dietz WH, Robinson TN. Use of the body mass index (BMI) as a measure of overweight in children and adolescents. J Pediatr 1998;132:191-193.Keys A, Fidanza F, Karvonen MJ, Kimura N, Taylor HL. Indices of relative weight and obesity. J Chronic Dis 1972;25:329-343.

Garrow JS. Treat obesity seriously: a clinical manual. Edinburgh:Churchill Livingstone, 1981.Glinski J, Wetzler S, Goodman E. The psychology of gastric bypass surgery. Obesity Surgery 2001;11:581-588.

American Psychiatric Association. Diagnostic and statistical manual of mental disorders, Fourth Edition, Text Revision. Washington, D.C.: American Psychiatric Association; 2000.

Wadden TA, Sarwer DB, Fabricatore AN, Jones L, Stack R, Williams NS. Psychosocial and behavioral status of patients undergoing bariatric surgery: What to expect before and after surgery. The Medical Clinics of North America 2007;91:451-469.

Stunkard AJ, Grace WJ, Wolff HG. The night-eating syndrome: A pattern of food intake among certain obese patients. American Journal of Medicine 1955;19:78-86.

Anderson DA, Wadden TA. Treating the obese patient: Archives of Family Medicine 1999;8:156-167.

Carr D, Friedman MA. Is obesity stigmatizing? Body weight, perceived discrimination, and psychological well-being in the United States. Journal of Health and Social Behavior 2005;46(3):244-259.

Herpertz, S, Kielmann R, Wolf AM, Langkafel, Senf W, Hebebrand, J. Does obesity surgery improve psychosocial functioning? A systematic review. International Journal of Obesity 2003;27:1300-1314.

Wing RR. Behavioral weight control. In: Wadden TA, Stunkard AJ. (Eds.) Handbook of obesity treatment. New York: Guilford Press; 2002.

[9] Cooper Z, Fairburn CG. A new cognitive behavioral approach to the treatment of obesity. Behaviour Research and Therapy 2001;39:499-511.

Kushner RF. Obesity management. Gastroenterology Clinics of North America 2007;36:191-210.

National Heart Lung and Blood Institute. The practical guide to identification, education and treatment of overweight and obesity in adults. NIH 2000 Pub no. 00-4084.

$\mathrm{NIH}$ Conference. Gastrointestinal surgery for severe obesity: Consensus development conference panel. Annals of Internal Medicine 1991;115:956-961.

van Hout GCM, Verschure SK, van Heck GL. Psychological predictors of success following bariatric surgery. Obesity Surgery 2005;15(4):552-560.

Pawlow LA, O'Neil PM, White MA, Byrne TK. Findings and outcomes of psychological evaluations of gastric bypass applicants. Surgery for Obesity and Related Diseases 2005;1:523-529.

Greenberg I, Perna F, Kaplan M, Sullivan MA. Behavioral and psychological factors in the assessment and treatment of obesity surgery patients. Obesity Research 2005;13(2):244-249. 
